\title{
MECHANISMS OF TOPICAL DELIVERY OF LIPOSOMALLY ENTRAPPED DRUGS*
}

\author{
N.F.H. Ho** \\ Drug Delivery Systems Research, The Upjohn Company, Kalamazoo, MI 49001 (U.S.A.) \\ M.G. Ganesan \\ Pharmaceutical Research and Development, Lederle Laboratories, Pearl River, NY 10965 (U.S.A.) \\ N.D. Weiner and G.L. Flynn \\ College of Pharmacy, The University of Michigan, Ann Arbor, MI 48109 (U.S.A.)
}

Our research on the mechanism by which liposomally entrapped solutes are transported across the skin was prompted by an investigation reported in the literature which concluded with meager supporting evidence that liposomes containing triamcinolone acetonide penetrated the stratum corneum intact and, thereby, increased skin absorption. To elucidate the mechanism we used glucose, hydrocortisone, progesterone and multilamellar DPPC liposomes. Experimental strategies involved: DSC determinations; in vivo permeation of hairless mouse skin by liposomes, by liposome-entrapped solutes (15:1) and by solutes in simple solution; and in vitro release kinetics of liposome-entrapped solutes.

The liposomes neither penetrated the skin nor fused with the stratum corneum. Progesterone and hydrocortisone, which were intercalated in the bilayer structure, permeated the skin with ease comparable to free drug. The skin transport of the highly polar glucose entrapped in the aqueous regions of the liposome was markedly slow as compared to the free species. Physical model analysis indicated that the slow release rate of glucose out of the liposome was the rate-determining step as compared to the relatively rapid skin permeation of the free solute. For the hydrophobic progesterone and hydrocortisone, quantitative analyses suggested direct transfer of drug from the liposome to the surface phases of skin and subsequent diffusion through the tissue. Considering this mechanism and owing to increased solubility of lipophilic drugs in liposomes, more total drug may be delivered through the skin by liposomes relative to simple aqueous solution.

\section{INTRODUCTION}

Liposomes have been investigated as a topical drug delivery system for the skin and eyes. Mechanistic studies are sparse. Our research on the mechanism by which liposomal-

\footnotetext{
* Paper presented at the Second International Sym posium on Recent Advances in Drug Delivery Systems, February 27, 28 and March 1, 1985, Salt Lake City, UT, U.S.A.

**To whom correspondence should be addressed.
}

ly entrapped solutes are transported across the skin was prompted by an investigation reported in the literature which concluded with meager supporting evidence that liposomes containing triamcinolone acetonide penetrated the stratum corneum intact and, thereby, increased skin absorption [2]. To elucidate the mechanism we used glucose, hydrocortisone and triamcinolone, multilamellar dipalmitoylphosphatidylcholine liposomes and hairless mouse skin [2].

0168-3659/85/\$03.30 $\odot 1985$ Elsevier Science Publishers B.V. 


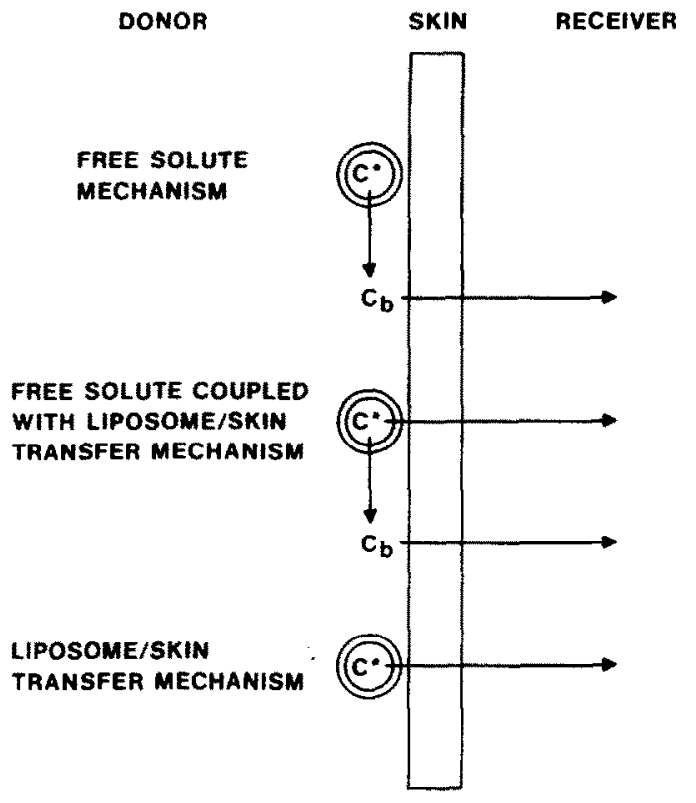

Fig. 1. Schematic description of various mechanisms in the skin permeation of liposome-entrapped solutes. Liposomes are not absorbed intact nor fused with the stratum corneum.

\section{THEORY}

The physical models considered in Fig. 1 include models for the following mechanisms: free solute mechanism; concurrent free solute and direct liposome/skin transfer mechanism; and direct liposome/skin transfer mechanism. The models assume that the liposomes are neither absorbed intact nor fused with the membrane.

\section{Free solute mechanism}

This mechanism involves the leakage of liposome-entrapped solute into the bulk aqueous medium and skin permeation of the free solute. The steady-state flux of free solute across the skin into the sink of the receiver compartment is

$J_{\mathrm{s}}=\left(\frac{1}{4 \pi a^{2} n P_{\mathrm{bl}}}+\frac{1}{A P_{\mathrm{s}}}\right)^{-1} C^{*}$

where $J_{s}=$ skin permeation rate, mass $/ \mathrm{h}$; $P_{\mathrm{bl}}=$ permeability coefficient of the solute for the liposome lipid bilayers, $\mathrm{cm} / \mathrm{h} ; P_{\mathrm{s}}=$ permeability coefficient of the skin (epidermis and dermis), $\mathrm{cm} / \mathrm{h} ; A=$ diffusional area of skin, $\mathrm{cm}^{2} ; n=$ total number of liposomes; $a=$ radius of a liposome, $\mathrm{cm}$; and $C^{*}=$ solute concentration in the liposomes.

Since the number of relatively monodispersed size liposomes is approximated by

$n=\frac{3 \epsilon V_{\mathrm{D}}}{4 \pi a^{3}}$

and the skin permeation rate is equal to ap. pearance rate

$J_{\mathrm{s}}=V \frac{\mathrm{d} C}{\mathrm{~d} t}$

where $\epsilon=$ volume fraction of liposomes; $V_{D}=$ volume of liposome suspension on donor side of the skin, $\mathrm{cm}^{3} ; V=$ volume of liquid on receiver side of $\mathrm{skin}, \mathrm{cm}^{3}$; and $C=$ permeant concentration on receiver side, mass $/ \mathrm{cm}^{3}$, it follows that the effective permeability coefficient, $P_{\mathrm{e}}(\mathrm{cm} / \mathrm{h})$, can be calculated by

$P_{\mathrm{e}}=\frac{V}{A C^{*}}\left(\frac{\Delta C}{\Delta t}\right)=\left(\frac{a A}{3 \epsilon V_{\mathrm{D}} P_{\mathrm{bl}}}+\frac{1}{P_{\mathrm{s}}}\right)^{-1}$

It is seen that, in extreme cases, the skin permeation rate will be rate-limited by the release of solute from the liposomes or the permeability of the skin itself. Experimentally, $P_{\mathrm{bl}}$ and $P_{\mathrm{s}}$ are directly accessible to quantitation by in vitro release studies from liposomes and skin permeation measurements of the solute in simple solution, respectively.

\section{Concurrent free solute and direct liposome/skin transfer mechanism}

In this model percutaneous absorption is ascribed to the sum of the permeation rate of the free solute released from the liposomes and rate involving direct transfer of solute between the liposome and skin followed by diffusion across the skin. The effective permeability coefficient is

$$
P_{\mathrm{e}}=\frac{V}{A C^{*}} \frac{\Delta C}{\Delta t}=P^{*}+\left(\frac{a A}{3 \epsilon V_{\mathrm{D}} P_{\mathrm{bl}}}+\frac{1}{P_{\mathrm{s}}}\right)^{-1}
$$


where $P^{*}$ is the effective permeability coefficient of the liposome/skin transfer and permeation processes. In the event $P^{*}$ is zero, eqn. (5) reduces to the expression for the free solute mechanism.

\section{Direct liposome/skin transfer mechanism}

When the release of a hydrophobic solute from liposomes is negligible but significant skin permeation occurs, then direct transfer of the solute between the liposomal bilayer and the lipid-like stratum corneum is a likely possibility. Hence

$P_{\mathrm{e}}=\frac{V}{A C^{*}}\left(\frac{\Delta C}{\Delta t}\right)=P^{*}$

\section{In vitro release kinetics}

By following the release kinetics of a liposomally entrapped solute in the bulk aqueous phase of the dispersion, the permeability coefficient of the bilayers, $P_{\mathrm{bl}}$, can be determined using the linear expression

$C_{\mathrm{b}}=C_{\mathrm{b}}(0)+\left(\frac{3 P_{\mathrm{bl}} T}{a(1-\epsilon) V}\right) t$

where $C_{\mathrm{b}}, C_{\mathrm{b}}(0)=$ solute concentrations in the bulk liquid at time $t$ and zero, respectively; $T=$ initial amount of liposome-entrapped solute; $\epsilon=$ volume fraction of liposomes; $V=$ volume of the suspension; and $a=$ radius of the liposomes. The equation is valid for the period in which the amount of solute released is small.

\section{EXPERIMENTAL}

The preparation details of multilamellar DPPC (dipalmitoylphosphatidylcholine) and liposomes containing cold and radiolabeled glucose, hydrocortisone and progesterone have been described previously [2]. Briefly, it involves the dispersion of a dry thin layer of lipids off the wall of a flask by vortex mixing with normal saline followed by sonication and successive washing and centrifugation to obtain a relatively narrow particle size distribution. The DPPC to solute molar ratio was about 15:1.

Experimental strategies involved: differential scanning calorimetry (DSC) determinations of liposome preparations; in vitro release kinetics of liposome-entrapped solutes; and in vitro skin permeation studies using blank liposomes, liposome-entrapped solutes and solutes in simple solution. Thermograms were obtained by DSC (Perkin-Elmer, model DSC-2C with data analysis module) on $1 \mu \mathrm{mol}$ lipid samples at a heating rate of $5^{\circ} \mathrm{C} / \mathrm{min}$. Release experiments of radiolabeled solutes from liposomes were carried out in a beaker at $37^{\circ} \mathrm{C}$ and the kinetics were determined by liquid scintillation counting of supernatant samples obtained over a $60 \mathrm{~h}$ period.

Using a Franz diffusion cell and excised hairless mouse skin, the appearance kinetics of radiolabeled liposome preparations and solutes in normal saline were followed at $37^{\circ} \mathrm{C}$ for $60 \mathrm{~h}$. The system is characterized by the following parameters: $A=0.785 \mathrm{~cm}^{2} ; V_{\mathfrak{D}}=$ $0.2 \mathrm{ml} ; V=5.2 \mathrm{ml} ; \epsilon=0.075$ as determined by measurement of the centrifuged plug; and $a=0.4 \mu \mathrm{m}$ ( 0.2 to $0.5 \mu \mathrm{m}$ range) estimated by negative stain electron microscopy.

\section{RESULTS AND DISCUSSION}

The pretransition and transition temperatures of the liposome preparations derived from DSC thermograms indicate that glucose is solely entrapped in the aqueous spaces of the multilamellar liposomes while the lipophilic steroids are predominantly intercalated in the lipid bilayers (Table 1). At $37^{\circ} \mathrm{C}$ the liposomes are in the so-called solid (gel) state. The ability of entrapped glucose, hydrocortisone and progesterone to diffuse out of the liposomes decreases with increasing lipophilicity. No detectable quantity of ${ }^{3} \mathrm{H}$ progesterone was found throughout the $60 \mathrm{~h}$ study. Estimates of the effective permeability coefficients of the lipid bilayers are found in Table 2. 
TABLE 1

Thermal properties of DPPC liposome systems

\begin{tabular}{lllll}
\hline System & \multicolumn{2}{l}{ Temperature, ${ }^{\mathrm{c}} \mathrm{C}$} & \multirow{2}{*}{ Log P.C. } & Comments \\
\cline { 2 - 4 } & Transition & Pretransition & & \\
\hline Blank DPPC liposome & 42 & 36 & - & - \\
Entrapped glucose $(12: 1)^{\mathrm{b}}$ & 42 & 36 & $<1.0$ & Entrapped in aqueous spaces \\
Entrapped hydrocortisone $(23: 1)$ & 39 & None & 1.53 & Intercalated in bilayer \\
Entrapped progesterone $(20: 1)$ & 41 & None & 3.99 & Intercalated in bilayer \\
\hline
\end{tabular}

${ }^{\mathrm{a}}$ Partition coefficient in n-octanol/water.

${ }^{b}$ Molar ratio of DPPC: solute.

TABLE 2

Summary of permeability coefficients

\begin{tabular}{lllll}
\hline System & $\begin{array}{l}\text { Permeability } \\
\text { coefficient, } \\
\mathrm{cm} / \mathrm{h}\end{array}$ & Glucose & Hydrocortisone & Progesterone \\
\hline $\begin{array}{l}\text { Skin permeation of } \\
\text { solutes in normal saline }\end{array}$ & $P_{\mathrm{s}}$ & $1.4 \times 10^{-3}$ & $5.4 \times 10^{-4}$ & $13.5 \times 10^{-4}$ \\
$\begin{array}{l}\text { Skin permeation of } \\
\text { liposome-entrapped solutes }\end{array}$ & $P_{\mathrm{e}}$ & $6 \times 10^{-9}$ & $4 \times 10^{-4}$ & $4.6 \times 10^{-4}$ \\
& $P_{\mathrm{bl}}$ & $4.2 \times 10^{-11}$ & - & $\sim 0$ \\
& $p^{*}$ & $\sim 0$ & $3.95 \times 10^{-4}$ & $4.6 \times 10^{-4}$ \\
& $P_{\mathrm{bl}}$ & $6.2 \times 10^{-9}$ & $3.7 \times 10^{-9}$ & $\sim 0$ \\
\hline $\begin{array}{l}\text { In vitro release from } \\
\text { liposomes }\end{array}$ & & & & 0 \\
\hline
\end{tabular}

The time course of the steady-state fractions of the applied amount of radiolabeled substances penetrating the skin are shown in Fig. 2. The absence of radiolabeled DPPC from blank liposomes and hydrocortisoneentrapped liposomes in the receiver side of the diffusion cell clearly indicates that the liposomes are incapable of diffusing intact through the dense stratum corneum. However, the liposomes appear to play various significant roles in delivering solutes to the skin surface for percutaneous absorption (Table 2). When glucose is entrapped in liposomes, its effective sk in permeability is found to be 2000 -fold smaller as compared to the case of glucose in normal saline. This observation gives additional supporting evidence that liposomes are not absorbed as intact entities, and also suggests the absence of fusion of liposomes with the stratum corneum. A quantitative, physical model analysis of the data reveals that a significant decrease in percutaneous absorption is expected when the release of glucose from the liposomes is the rate-determining step in the free solute mechanism. The permeability coefficient of the liposomal lipid bilayers, $P_{\mathrm{bl}}$, is $6.2 \times 10^{-9}$ $\mathrm{cm} / \mathrm{h}$ from in vitro release studies and com- 


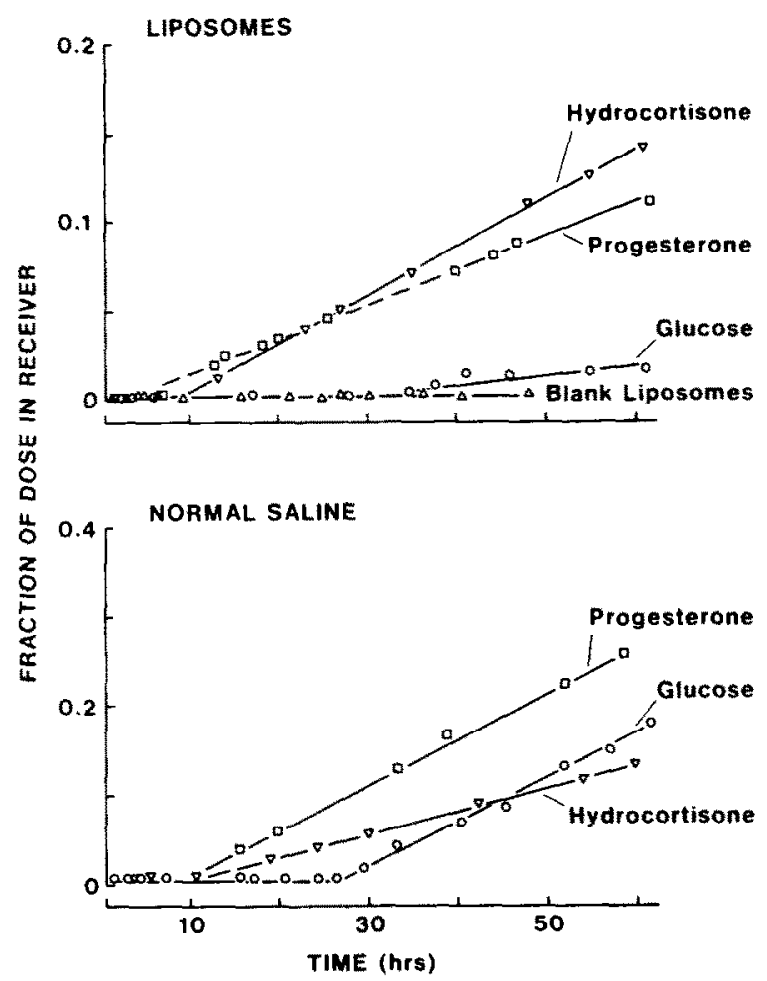

Fig. 2. Fraction of various permeants appearing in the receiver compartment with time from solutes in normal saline and liposomes.

pares reasonably well with $4.2 \times 10^{-10} \mathrm{~cm} / \mathrm{h}$ calculated by eqn. (4) using the skin permeation data.

In contrast to the glucose situation, the liposomally entrapped hydrocortisone and progesterone and these steroids in simple aqueous solution are transported across the skin with nearly the same efficiency. Because the liposomal release rates of these steroids into the bulk liquid are negligible, the direct transfer of the steroids between the lipid bilayer and the lipid phase of the stratum corneum followed by diffusion through the skin is alternatively proposed. This mechanism is reasonable in view of the collision complex transfer phenomenon between liposome-entrapped cholesterol and $\beta$-sitosterol and silicone rubber membrane studied by Kreuter et al. [3].

\section{CONCLUSION}

In this study the liposomes neither diffused across nor fused with the stratum corneum of the skin. From this perspective, liposomes do not provide a unique, mechanistic advantage in delivering drugs across the skin.

The fact that the slow release rate of glucose out of the liposomes was the ratedetermining step as compared to the relatively rapid skin permeation of the free solute in the overall absorption process suggests that, in general, the skin transport of highly polar compounds entrapped in the aqueous regions of liposomes will be markedly slow in contrast to simple solutions of the drug.

In the case of hydrocortisone and progesterone which are located in the lipid bilayers and do not readily leak out, the liposomeentrapped steroids permeated the skin with comparable facility to that of the free drug in solution. The mechanism of direct transfer of the steroids from the liposome to the surface phases of the skin and subsequent diffusion through the tissue was suggested. Considering this mechanism and owing to increased solubility of lipophilic drugs in liposomes, more total drug may be delivered through the skin via liposomes relative to simple aqueous solution.

\section{REFERENCES}

1 M. Mezei and V. Gulasekharam, Liposomes - A selective drug delivery system for the topical route of administration. I. Lotion dosage form, Life Sci., 26 (1980) 1473-1477.

2 M.G. Ganesan, N.D. Weiner, G.L. Flynn and N.F.H. Ho, Influence of liposomal entrapment on percutaneous absorption, Int. J. Pharm., 20 (1984) 139-154.

3 J. Kreuter, W.I. Higuchi, M.G. Ganesan and N.D. Weiner, Delivery of liposome membrane-associated sterols through silastic membranes, Biochim. Biophys. Acta, 676 (1981) 118-121. 